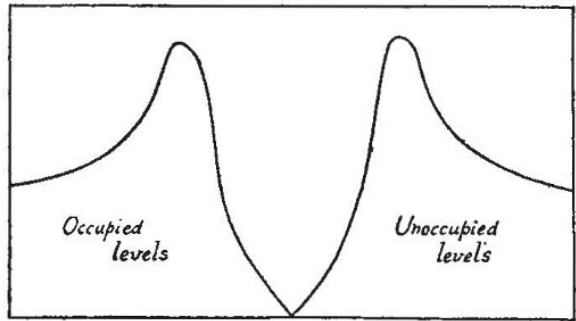

electrons get into the upper half, but only in small numbers, and conduction is possible. Such conduction increases with temperature. All this is in agreement with known experimental behaviour.

Our calculations, which will be reported more fully elsewhere, have been extended to include the previously neglected overlap integral, and to take account of interaction between the separate graphite planes. The general results described above are not significantly altered.

Physical Chemistry Laboratory,

\section{A. Coulson}

Oxford. Dec. 25.

${ }^{2}$ Lennard-Jones, J. E., Trans. Faraday Soc., 30, 58 (1934).

Coulson, C. A., Nature, 154, 797 (1944).

' Lennard-Jones, J. E., and Coulson, C. A., Trans. Faraday Soc., 35, 811 (1939).

'Bradburn, M., Coulson, C. A., and Rushbrooke, G. S., Proc. Roy. Soc. Edin., in the press.

\section{Raman Spectrum of Rock-Salt Under High Resolution}

THE original investigation of Rasetti ${ }^{1}$ as well as my later work ${ }^{2}$ on the Raman effect in rock-salt were carried out using a Hilger medium-size quartz spectro. graph for recording the faint second-order spectrum. In view of the fundamental importance of the case, it appeared desirable to re-investigate the subject, using the largest possible resolution and dispersion. Various improvements in experimental technique, including especially the efficient filtering of the exciting radiation before its entry into the spectro. graph, steady running conditions of the are and the temperature control of the spectrograph, have enabled me to work with the larger Hilger El quartz spectrograph for recording the Raman spectrum. Using a fine slit which necessitated an exposure of 160 hours, a record was obtained in which the details are not sensibly obscured by halation and which exhibits the true nature of the spectrum unambiguously.

It will be seen from the reproduction that the spectrum exhibits a set of discrete Raman lines clearly resolved from each other. The positions of the nine prominent lines with their frequency shifts in wave-numbers from the exciting line are indicated above the reproduction. The line with the frequency shift of $235 \mathrm{~cm}^{-1}$, to the sharpness of which Rasetti ${ }^{1}$ directed attention in his original investigation, con. tinues to be sharp even under high resolution. The width of this line does not exceed 3 wave-numbers if due allowance is made for the effect of instrumental and photographic factors. Its intensity is more than ten times that of the background on either side. On the longer wave-length side of this prominent line, there is an exceedingly fine line with the frequency shift of $258 \mathrm{~cm}^{-1}$ which is clearly discernible against the background. The intense lines with frequency shifts 300 and $314 \mathrm{~cm}^{-1}$ are also fairly sharp. The pairs of lines with frequency shifts

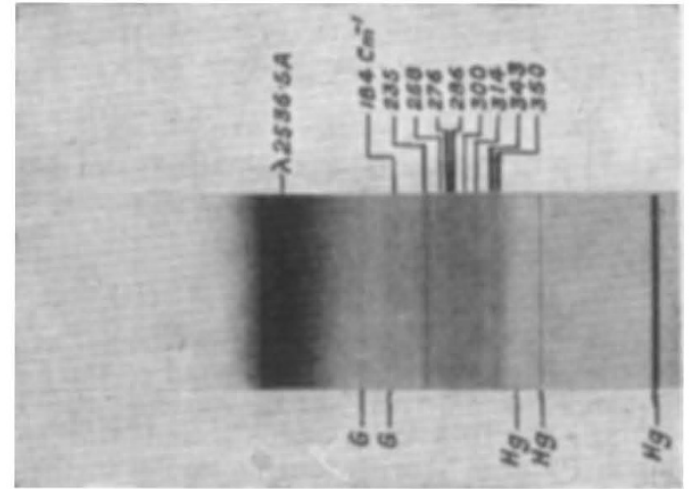

RAMAN SPECTRUM OF ROCK-SALT TAKEN WITH A HILGER E1 QUARTZ SPECTROGRAPH

$276,286 \mathrm{~cm} .^{-1}$ and $343,350 \mathrm{~cm} .^{-1}$ have a finite width, but small compared with the width of the peaks in the BornBradburn theoretical intensity distribution curve ${ }^{3}$.

It may be remarked that in many crystals, for example, diamond 4 , quart $z^{5}$, calcite ${ }^{6}$, etc., the Raman lines which appear extremely sharp at ordinary temperatures broaden to varying extents at higher temperatures. It may therefore be presumed that in the case of rock-salt as well, the observed differ. ences in width of the various lines at room tempera. ture are due to thermal agitation in the crystal. It appears also not unlikely that if the spectrum could be successfully recorded with the crystal held at liquid air temperature, all the lines would appear extremely sharp.

The main conclusion that emerges from the present investigation is that the vibration spectrum of the rock-salt lattice in the infra-red region of frequencies as manifested in the second-order Raman effect is a discrete one, and is different in its nature from that deduced from the lattice dynamics of Born. In view of the results of the present experimental investigation, it does not appear necessary for me to offer any further comments on the points raised by Born? in his recent communication in Nature.

Physics Department,

Indian Institute of Science, Bangalore. Dec. 14.

1 Rasetti, F., Nature, 127, 626 (1931). Krishnan, R. S., Nature, 166, $267(1945) ; 157,623$ (1946); Proo
Ind. Acad. Sci., A, 187, 567 (1946).

- Born, M., and Bradburn, M., Nature, 156, 567 (1945).

- Krishnan, R. S., Proc. Ind. Acad. Sci., A, 24, 45 (1946).

- Nedungadi, T. M. K., Proc. Ind. Acad. Sci., A, 11, 86 (1940).

- Ornstein and Went, Physica, 2, 503 (1935).

' Born, M., Nature, 157, 810 (1946).

THE new photograph of the Raman spectrum of rock-salt seems to me no improvement on previous work, either on that of Rasetti or of Krishnan him. self. The dispersion appears to be larger, but not the resolution, so far as it is possible to judge from the reproduction. As no photometric curve is given, Krishnan's contentions about the breadth of the 'lines' are without foundation.

A complete account of the theory by Miss Brad. burn and myself has meanwhile appeared ${ }^{1}$. It does not claim to explain all details of the spectrum, because of the simplifications and approximations which were necessary to make the problem tractable. For example, we have replaced the thirty-six branches of the rigorous formula by eighteen, omitting some 